\title{
The sub-nucleolar localization of PHF6 defines its role in rDNA transcription and early processing events
}

\author{
Matthew AM Todd ${ }^{1,2}$, Michael S Huh ${ }^{1}$ and David J Picketts ${ }^{\star, 1,2,3}$
}

Ribosomal RNA synthesis occurs in the nucleolus and is a tightly regulated process that is targeted in some developmental diseases and hyperactivated in multiple cancers. Subcellular localization and immunoprecipitation coupled mass spectrometry demonstrated that a proportion of plant homeodomain (PHD) finger protein 6 (PHF6) protein is localized within the nucleolus and interacts with proteins involved in ribosomal processing. PHF6 sequence variants cause Börjeson-Forssman-Lehmann syndrome (BFLS, MIM\#301900) and are also associated with a female-specific phenotype overlapping with Coffin-Siris syndrome (MIM\#135900), T-cell acute lymphoblastic leukemia (MIM\#613065), and acute myeloid leukemia (MIM\#601626); however, very little is known about its cellular function, including its nucleolar role. HEK 293T cells were treated with RNase A, DNase I, actinomycin D, or 5,6-dichloro- $\beta$-D-ribofuranosylbenzimadole, followed by immunocytochemistry to determine PHF6 sub-nucleolar localization. We observed RNA-dependent localization of PHF6 to the sub-nucleolar fibrillar center (FC) and dense fibrillar component (DFC), at whose interface rRNA transcription occurs. Subsequent ChIP-qPCR analysis revealed strong enrichment of PHF6 across the entire rDNA-coding sequence but not along the intergenic spacer (IGS) region. When rRNA levels were quantified in a PHF6 gain-of-function model, we observed an overall decrease in rRNA transcription, accompanied by a modest increase in repressive promoter-associated RNA (pRNA) and a significant increase in the expression levels of the noncoding IGS 36 RNA and IGS ${ }_{39}$ RNA transcripts. Collectively, our results demonstrate a role for PHF6 in carefully mediating the overall levels of ribosome biogenesis within a cell.

European Journal of Human Genetics (2016) 24, 1453-1459; doi:10.1038/ejhg.2016.40; published online 11 May 2016

\section{INTRODUCTION}

Sequence variants of the X-linked gene coding for the plant homeodomain (PHD) finger protein 6 (PHF6) are the only known cause of Börjeson-Forssman-Lehmann syndrome (BFLS, MIM\#301900), ${ }^{1}$ for which common features include intellectual disability, truncal obesity, gynaecomastia, hypogonadism, large ears, coarse facial features, and digit abnormalities. ${ }^{2}$ More recently, PHF6 sequence variants were also linked to T-cell acute lymphoblastic leukemia (T-ALL), acute myeloid leukemia, and a female-specific disorder that closely overlaps with Coffin-Siris syndrome. ${ }^{3-5}$ Although cancer has only been reported in $6.5 \%$ of BFLS patients, ${ }^{6-8}$ a recent study showed PHF6 loss to reduce growth rates of B-cell ALL tumors, ${ }^{9}$ indicative of a context-dependent requirement for PHF6 in cells.

Structurally, PHF6 contains both nuclear and nucleolar localization sequences, and two ZaP domains (Zinc knuckle, atypical PHD), ${ }^{10}$ the second of which interacts with double-stranded DNA. ${ }^{11}$ Although the specific functions of PHF6 remain unclear, we have previously shown PHF6 to co-purify with multiple constituents (CHD4, RBBP4, HDAC1) of the nucleosome remodeling and deacetylation (NuRD) complex (Peptide Atlas: PASS00849), which regulates gene expression during embryogenesis and lineage commitment. ${ }^{10,12}$ These findings were validated by Liu et al, ${ }^{11}$ who confirmed a direct interaction between PHF6 and RBBP4. Other studies have highlighted transcriptional roles for PHF6, including interactions with PAF1, which affects transcriptional elongation of gene targets that mediate neuronal migration, ${ }^{13}$ and with upstream binding factor UBF, a master transcriptional activator of rRNA. ${ }^{13,14}$ Moreover, our own proteomic screen identified interactions between PHF6 and ribosomal proteins involved in rRNA processing. ${ }^{10}$ Considering the significant proportion of PHF6 protein that resides in the nucleolus, ${ }^{10}$ it is possible that PHF6 may be an important contributor toward ribosome biogenesis.

Ribosomal output is directly correlated with nucleolar size, with large nucleoli linked to poor cancer prognoses. ${ }^{15}$ Indeed, several ribosomal protein genes (eg, RPL5, RPL10, RPL11, RPL22) are mutated in T-ALL, ${ }^{16}$ and nucleolar dysregulation is linked with intellectual disability (eg, Cockayne syndrome) and other neurological disorders (eg, Alzheimer's disease, Parkinson's disease). ${ }^{17,18}$ Nucleoli are sub-nuclear compartments that form around nucleolar organizer regions at ribosomal DNA (rDNA) tandem repeats. Each repeat of the 43-kb rDNA gene (GenBank: U13369.1) consists of a 13-kb coding sequence for the polycistronic $47 \mathrm{~S}$ pre-rRNA transcript and a $30-\mathrm{kb}$ intergenic spacer (IGS) region. During ribosome biogenesis, $47 \mathrm{~S}$ prerRNA is transcribed and processed into $18 \mathrm{~S}, 5.8 \mathrm{~S}$, and $28 \mathrm{~S}$ rRNA and then incorporated into $60 \mathrm{~S}$ and $40 \mathrm{~S}$ ribosomes. ${ }^{19}$ These processes are carried out in three sub-compartments (Figure 1a): the fibrillar center (FC), where rDNA is located; the dense fibrillar component (DFC), for early rRNA processing; and the granular component (GC), for late rRNA processing, with pre-rRNA transcription occurring at the FC/ DFC interface. ${ }^{20,21}$ In addition to UBF, both NuRD and PAF1 have been demonstrated to mediate RNA Pol I-dependent rRNA

\footnotetext{
${ }^{1}$ Regenerative Medicine Program, Ottawa Hospital Research Institute, Ottawa, Ontario, Canada; ${ }^{2}$ Department of Biochemistry, Microbiology, and Immunology, Faculty of Medicine, University of Ottawa, Ottawa, Ontario, Canada; ${ }^{3}$ Department of Cellular and Molecular Medicine, Faculty of Medicine, University of Ottawa, Ottawa, Ontario, Canada ${ }^{*}$ Correspondence: Dr DJ Picketts, Regenerative Medicine Program, Ottawa Hospital Research Institute, Room C4419, 501 Smyth Road, Ottawa, Ontario K1H 8L6, Canada. Tel: +1 (613) 737 8989; Fax: +1 (613) 737 8803; E-mail: dpicketts@ohri.ca

Received 19 January 2016; revised 30 March 2016; accepted 12 April 2016; published online 11 May 2016
} 
a

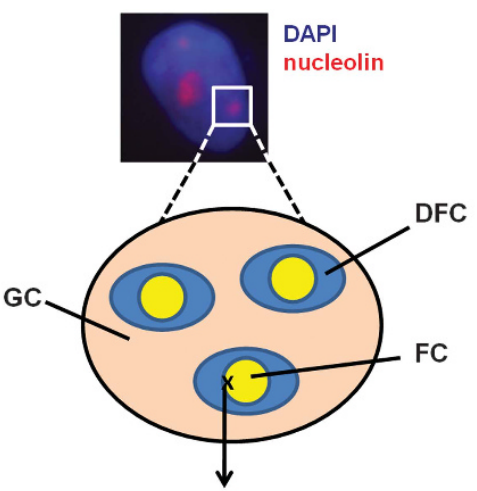

RNA pol I-mediated transcription of rDNA repeats

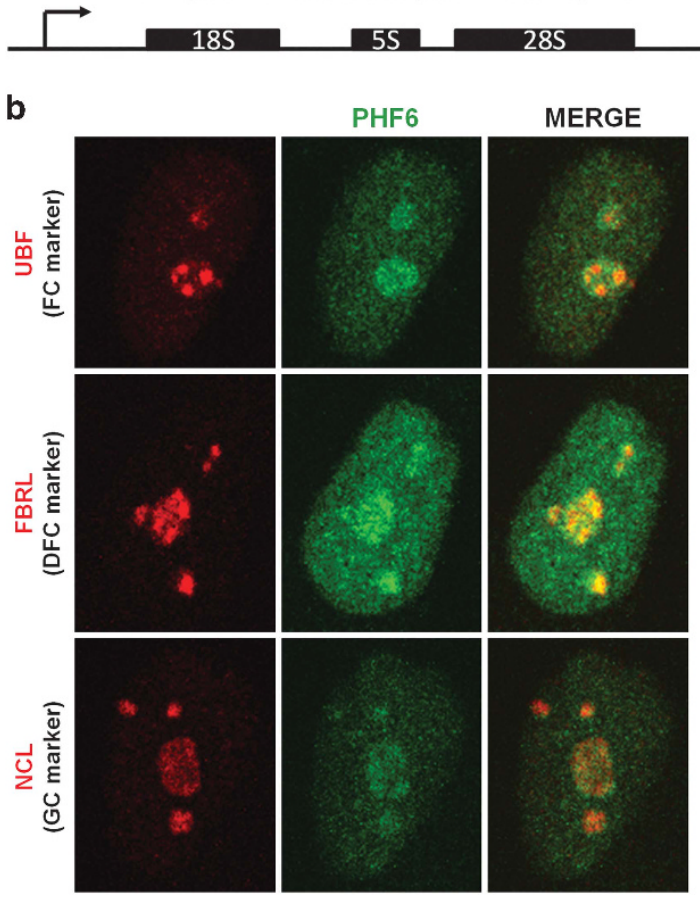

Figure $1 \mathrm{~A}$ subset of nuclear PHF6 localizes to the nucleolus. (a) Schematic representation of the three sub-nucleolar compartments: the fibrillar center $(F C)$, the dense fibrillar component (DFC), and the granular component (GC). The image depicts the cell nucleus counterstained with DAPI (blue) and the nucleoli visualized by $\mathrm{NCL}$ staining (red). (b) Representative images of immunofluorescently labeled of HeLa cells with antibodies to PHF6 (green) and specific markers (red) for the FC (UBF), DFC (FBRL), and GC (NCL).

transcription..$^{22,23}$ Therefore, further studies are warranted to fully dissect the specific nucleolar responsibilities with which PHF6 is tasked.

The objective of this study was to utilize sub-nucleolar fractionation to elucidate the role of PHF6 in regulating rDNA expression. We observed nucleolar PHF6 to be localized within both the FC and DFC, where it binds to rDNA-coding sequences. We also report the novel findings that PHF6 is recruited to the nucleolus in an RNA-dependent manner and that PHF6 overexpression modestly reduces the expression of rRNA transcripts, coinciding with elevated levels of non-coding $\mathrm{IGS}_{36} \mathrm{RNA} \mathrm{IGS}_{39} \mathrm{RNA}$, and promoter RNA (pRNA) transcripts. Overall, our findings confirm a specific role for PHF6 in modulating the expression of multiple transcripts from rDNA repeats.

\section{MATERIALS AND METHODS}

\section{Cell culture and chemical treatments}

HeLa and HEK 293T cells were cultured at $37{ }^{\circ} \mathrm{C}$ in DMEM with $10 \%$ FBS and $1 \%$ penicillin-streptomycin. For chemical treatment, either, $0.5 \mu \mathrm{g} / \mathrm{ml}$ actinomycin D (ActD) (2 h; Sigma, Oakville, ON, Canada), $25 \mu \mathrm{g} / \mathrm{ml} 5,6$-dichloro- $\beta$ D-ribofuranosylbenzimadole (DRB) $(2 \mathrm{~h}$; Sigma), $5 \mu \mathrm{g} / \mathrm{ml} \alpha$-amanitin $(2 \mathrm{~h}$; Sigma), $10 \mathrm{ng} / \mathrm{ml}$ trichostatin A (TSA) (2 h; Sigma) or $50 \mu \mathrm{M} 5$-azacytidine (5azaC) ( $2 \mathrm{~h}$; Sigma) were added prior to harvesting cells for immunocytochemistry (ICC) or RNA. As a control, some DRB treatments were grown for an extra $2 \mathrm{~h}$ in DRB-free media (Supplementary Figure S1A). HEK 293T stable cell lines expressing pBRIT-LoxP-PHF6-NTAP or pBRIT-LoxP-PHF6-CTAP were grown in media with $1.5 \mu \mathrm{g} / \mu \mathrm{l}$ puromycin (Sigma). The sub-cloning of PHF6 cDNA (NCBI RefSeq: NM_032458.2) into the pBRIT-LoxP vectors (17520 Addgene, Cambridge, MA, USA) has been previously described. ${ }^{10}$

\section{Immunocytochemistry}

Cell fixation and ICC were performed as previously described. ${ }^{10}$ Primary and secondary antibodies, and conditions, are listed in Supplementary Table S1.

\section{Nuclease treatments}

Nuclease treatments were adapted from the protocol described by Chamousset et al. ${ }^{24}$ Briefly, slides containing HEK 293T ( 15000$)$ cells were permeabilized with $0.1 \%$ Triton X-100 in ASE buffer (20 mM Tris pH 7.5, $5 \mathrm{~mm} \mathrm{MgCl}_{2}$, $0.5 \mathrm{~mm}$ EGTA) and then incubated ( $20 \mathrm{~min}$, room temperature) with $10 \mu \mathrm{g} / \mathrm{ml}$ DNase I or $100 \mu \mathrm{g} / \mathrm{ml}$ RNase A. Untreated controls were incubated in nucleasefree ASE buffer. ICC was performed in combination with DAPI $(100 \mathrm{ng} / \mathrm{ml})$ and Pyronin Y $(10 \mu \mathrm{M})$ stains.

\section{Image acquisition and processing}

All cell images were captured using a Zeiss LSM 510 laser scanning confocal microscope (Zeiss, Goettingen, Germany), equipped with UV (405 nm), argon $(488 \mathrm{~nm})$, and helium/neon $(546 \mathrm{~nm})$ lasers. Images were initially analyzed with the Zeiss ZEN 2009 software (Zeiss, Munich, Germany) and then exported to the Zeiss LSM Image Browser v.4.2.0.121 software (Zeiss, Munich, Germany) for contrast processing.

\section{Chromatin immunoprecipitation (ChIP)}

HEK 293 T cells were crosslinked ( $1 \%$ formaldehyde, $10 \mathrm{~min}$ ) and chromatin sheared by probe sonication $(5 \times 10 \mathrm{~s}$ pulses, $30 \%$ amplitude), to an average fragment size of 400-500 bp. Chromatin from $10^{6}$ cells was immunoprecipitated using the EZ-ChIP protocol (No. 17-371 Millipore, Etobicoke, ON, Canada) with $1 \mu \mathrm{g}$ of antibody (see Supplementary Table S1) and then captured with $60 \mu \mathrm{l}$ preblocked Protein A-sepharose (GE Healthcare, Mississauga, ON, Canada), Protein G-agarose (Santa Cruz, Dallas, TX, USA), or M2-Flag beads (Sigma; Supplementary Figure S3D). Eluted products were treated with $1 \mu \mathrm{g}$ RNase A and $1 \mu \mathrm{g}$ proteinase $\mathrm{K}$ prior to purification by phenol-chloroform extraction and ethanol precipitation.

\section{RNA extraction and quantitative real-time PCR}

RNA from $10^{6}$ cells was extracted using an RNeasy Mini Kit (Qiagen, Toronto, ON, Canada), and then $1 \mu \mathrm{g}$ of total RNA was reverse transcribed (RT) with random primers and SuperScript III enzyme (Life Technologies, Burlington, ON, Canada). qPCR for ChIP-DNA or RT samples was prepared using SYBR Green Advantage qPCR premix (Clontech, Mountain View, CA, USA), and reactions were analyzed on an Mx3000P system (Agilent Technologies Canada Inc., Mississauga, ON, Canada). All primer sets are listed in Supplementary Table S2.

\section{Statistics}

Statistical analyses were performed in Microsoft Excel (Microsoft Corporation, Redmond, WA, USA), with means and SE calculated for either raw Ct (ChIPqPCR) or dCt values (qRT-PCR). For ChIP-qPCR, rDNA fold changes between ChIP products and $1 \%$ input were calculated using: fold change $=2^{\text {(input mean Ct-ChIP mean Ct) }}$. For qRT-PCR, fold changes were calculated with the $\Delta \Delta \mathrm{Ct}$ method using GAPDH as an internal control. Fold changes were calculated for means with and without SE. Significance was 
determined by two-tailed $t$-tests of unequal variance ( $95 \%$ and $99 \%$ confidence intervals).

\section{RESULTS}

PHF6 localizes to the nucleolar FC and DFC

To understand the nucleolar role of PHF6, we first defined its subnucleolar localization (see Figure 1b) in HeLa cells using the following markers: UBF (a DNA-dependent FC marker), fibrillarin (FBRL, an RNA-dependent DFC marker), or nucleolin (NCL, an RNAdependent GC marker). We observed some overlap of PHF6 with each marker, yet the study was inconclusive in identifying a specific compartment within which PHF6 resides.

Previous reports show that proteins co-localized within particular sub-compartments remain associated during nucleolar reorganization (Figure 2a), ${ }^{25-27}$ therefore we postulated that the same principle might apply to PHF6 co-localization with sub-nucleolar markers. In this regard, cells chemically treated with ActD induce nucleolar disassembly by inhibiting RNA Pol I, thereby arresting pre-rRNA transcription, while DRB inhibits casein kinase 2 to uncouple the rRNA processing machinery from RNA Pol I-mediated transcription. ${ }^{24}$ To further delineate PHF6 localization within the nucleolus, HEK 293T cells were treated with $0.5 \mu \mathrm{g} / \mathrm{ml} \mathrm{ActD}$ or $25 \mu \mathrm{g} / \mathrm{ml} \mathrm{DRB}$ for $2 \mathrm{~h}$ prior to immunocytochemistry (ICC) and confocal microscopy, using untreated cells as a negative control (Figures $2 \mathrm{~b}-\mathrm{d}$ ). Following ActD or DRB treatments, the bulk of the PHF6 foci co-localized with UBF (Figure 2b) and partially co-localized with FBRL (Figure 2c), but no distinct overlap was apparent between PHF6 and NCL (Figure 2d). Because ActD (at higher concentrations) and DRB are known to inhibit RNA Pol II, ${ }^{28,29}$ we also treated HEK 293T cells with $\alpha$-amanitin $(5 \mu \mathrm{g} / \mathrm{ml}, 2 \mathrm{~h})$, which specifically inhibits RNA Pol II but not RNA Pol I. ${ }^{30}$ As shown in Supplementary Figure S1A, $\alpha$-amanitin treatment did not induce nucleolar reorganization. In addition, the a
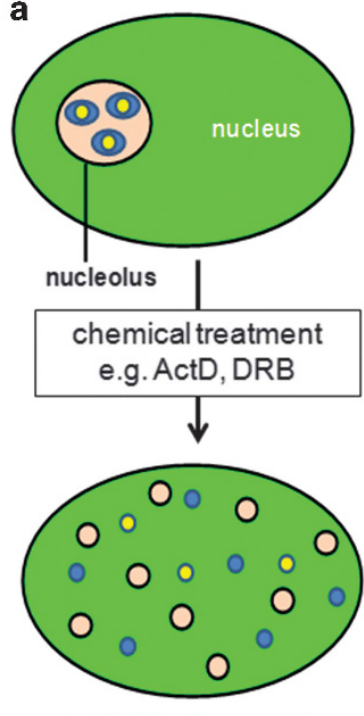

nucleolar disassembly b
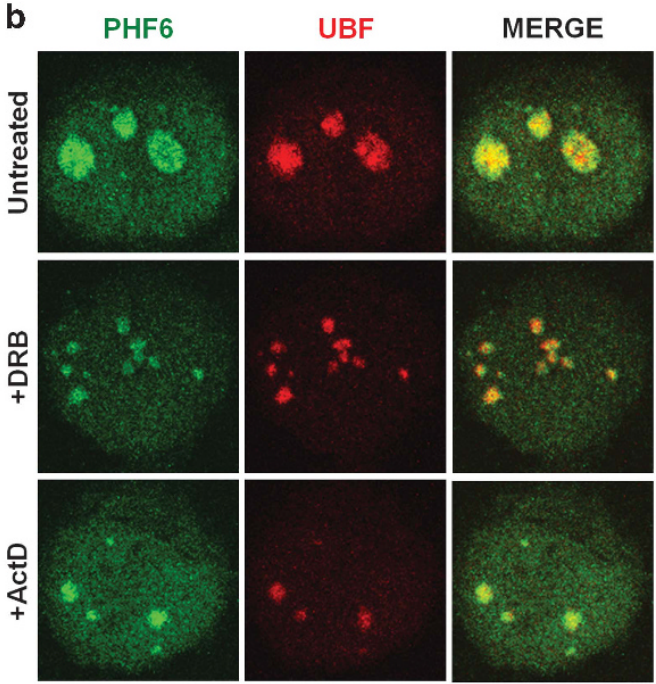

d

C PHF6
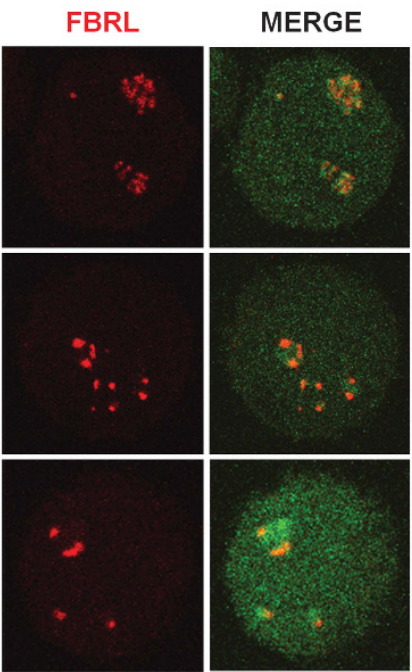

NCL

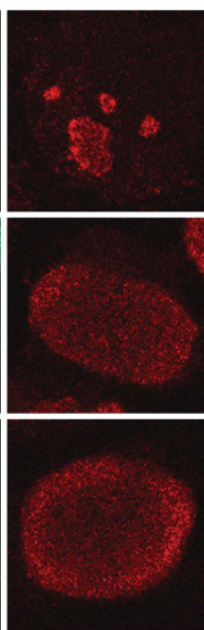

MERGE

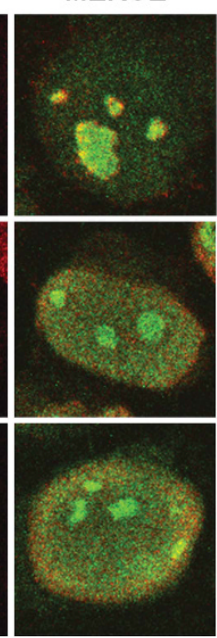

Figure 2 PHF6 co-localizes with the FC and partially co-localizes with the DFC. (a) Nucleoli can be partially or completely disassembled in response to chemical treatment with Act D or DRB. During nucleolar reorganization, proteins that are found in the same nucleolar compartments commonly remain associated in the same nascent foci. HEK 293T cells were treated with $25 \mu \mathrm{g} / \mathrm{ml} \mathrm{DRB}$ or $0.5 \mu \mathrm{g} / \mathrm{ml}$ Act D for $2 \mathrm{~h}$ and then fixed and labeled with antibodies to PHF6 (green) and (b) UBF, (c) FBRL, or (d) NCL (red). Representative cell images are shown. See Supplementary Figure S1 for additional controls. 
effects of DRB are completely reversible upon its removal. ${ }^{28}$ As such, some cells were treated with $25 \mu \mathrm{g} / \mathrm{ml} \mathrm{DRB}$ for $2 \mathrm{~h}$ and then grown in untreated media (without DRB) for a further $2 \mathrm{~h}$ to assess the reversibility of the DRB-induced nucleolar disassembly. Indeed, the DRB-induced PHF6 reorganization proved to be reversible (Supplementary Figure S1A).

Cajal bodies exist as sites for snRNP/snoRNP biogenesis and are recruited to nucleoli in response to DRB exposure as well as other instances of nucleolar stress. ${ }^{31}$ To determine whether PHF6 associates with Cajal bodies, we co-labeled untreated or treated cells with PHF6 and COILIN. Although PHF6 did not associate with Cajal bodies in the untreated cells, we did observe a partial overlap between PHF6 and COILIN for both the ActD and DRB treatments (Supplementary Figure S1B). Thus, from these experiments, we conclude that PHF6 is most likely localized to the FC/DFC interface.

PHF6 localization to the nucleolus is RNA dependent

The FC/DFC interface, where 47S pre-rRNA is transcribed from rDNA, harbors proteins with the ability to interact with both DNA and RNA. Recent literature indicates that PHF6 binds dsDNA ${ }^{11}$ and is recruited to the rDNA promoter, ${ }^{14}$ so we expected nucleolar recruitment of PHF6 to be DNA dependent. To test this hypothesis, HEK 293T cells were permeabilized and treated with DNase I or RNase A prior to fixation, and then ICC was performed for PHF6 and either
UBF (DNA-dependent marker) or nucleolin (RNA-dependent marker) (Figures $3 \mathrm{a}$ and $\mathrm{b}$ ). As a transcriptional activator, UBF directly binds to the transcriptional start site and coding regions of rDNA. ${ }^{21}$ As expected, DNase I treatment resulted in the loss of UBF from the nucleolus, but RNase A had no effect (Figure 3a), while nucleolin localization was dependent on RNA but not on DNA (Figure 3b). DNA loss with DNase I, but not with RNase A, was confirmed by DAPI staining (Figure 3a). Likewise, RNA loss with RNase A, but not with DNase I, was confirmed by Pyronin Y staining (Supplementary Figure S2A). To our surprise, PHF6 remained in the nucleolus after DNase I treatment (Figures $3 \mathrm{a}$ and $\mathrm{b}$ ) but was lost with RNase A, suggesting that PHF6 mainly associates with the nucleolus through an RNA-based mechanism. Fibrillarin was also significantly depleted in nucleoli following RNase A treatment but not DNase I (Supplementary Figure S2B). Taken together, we conclude that PHF6 requires an association with RNA in order to localize to the nucleolus. As fibrillarin associates with both rDNA and $\mathrm{rRNA}^{32}$ but was only depleted with RNase A in our hands, we cannot exclude the possibility that PHF6 interacts with both DNA and RNA.

\section{PHF6 binds to the rDNA-coding sequence}

PHF6 localization to the FC and DFC strongly implies a role for PHF6 in rRNA transcriptional regulation. As such, we performed ChIPqPCR with previously described primer pairs ${ }^{33}$ to determine whether:
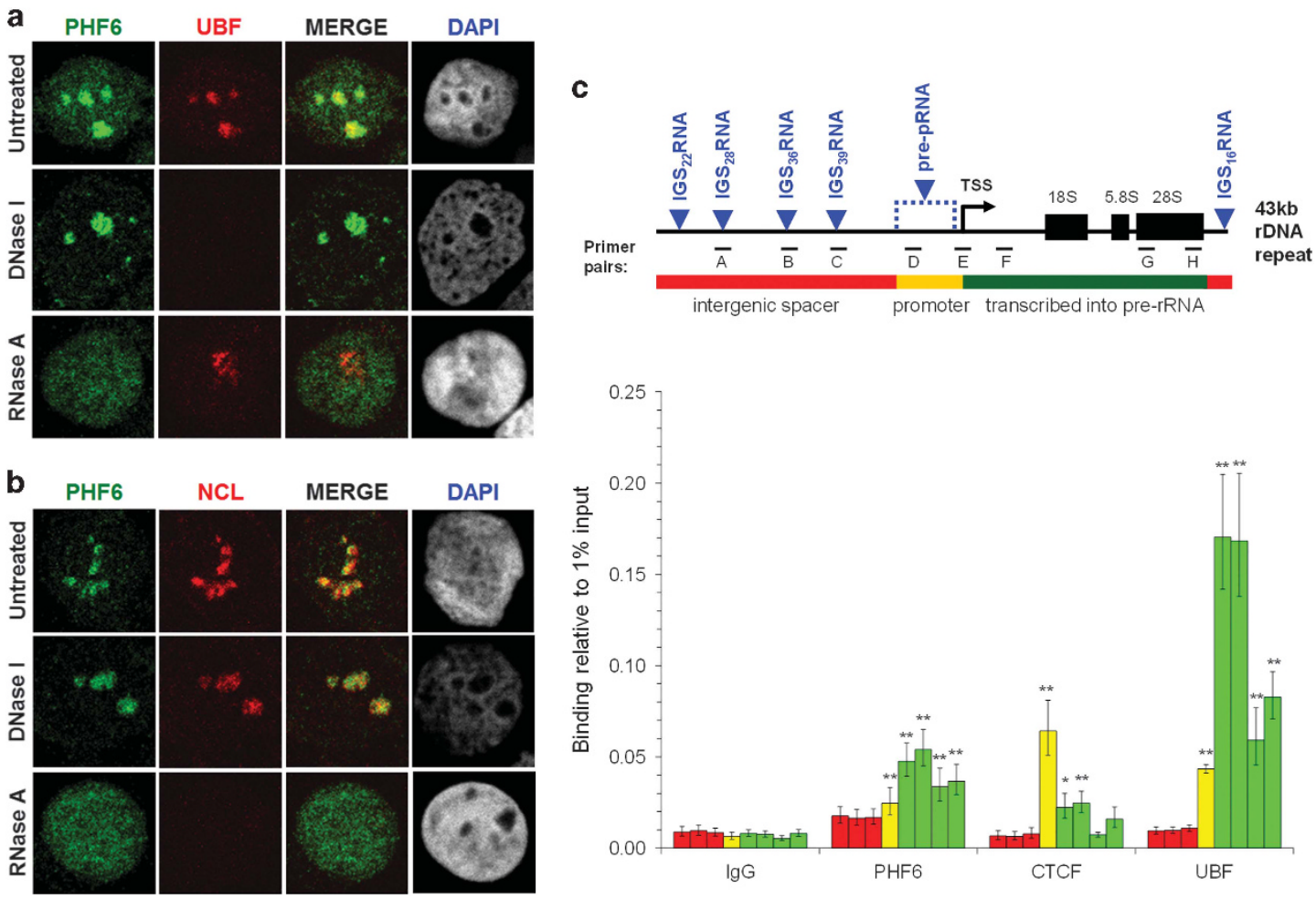

Figure 3 PHF6 localizes to the nucleolus in an RNA-dependent manner, where it binds to rDNA-coding sequences. (a, b) HEK 293T cells were treated with DNase I $(10 \mu \mathrm{g} / \mathrm{ml})$ or RNase A $(100 \mu \mathrm{g} / \mathrm{ml})$ and then immunofluorescently stained with antibodies to PHF6 (green) and UBF (A; red) or NCL (B; red). Representative cell images are shown. (c) Schematic diagram of the rDNA gene repeat (GenBank: U13369.1), showing the intergenic spacer (IGS) region (red), the rDNA promoter (yellow), and the rRNA-coding region (green). Primer pairs were designed to amplify IGS sequences (A: -15634/-15523; B: $-6942 /-6839 ; \mathrm{C}:-3712 /-3610)$, the promoter sequence (D: $-1017 /-924)$, and the coding sequence $(E:-47 /+32 ; F:+307 /+445 ; G:+8204 /$ +8300; $\mathrm{H}:+12855 /+12970$ ). The locations of sequences corresponding to non-coding IGS and unprocessed pRNA (pre-pRNA) transcripts are indicated in blue. PHF6 enrichment throughout the rDNA repeat was determined by ChIP-qPCR $(n=18)$ analysis performed from approximately $10^{6}$ HEK 293 T cells (per experiment). CTCF $(n=9)$ and UBF $(n=13)$ ChIP-qPCR products were quantitated as controls for binding to the coding rDNA promoter and coding sequence, respectively. For each antibody, the individual bars on the graph represent the binding (relative to $1 \%$ input) for individual primer sets in sequential order: IGS (primer sets A-C, red), promoter (primer set D, yellow), and coding sequence (primer sets E-H, green). See Supplementary Figure S2 for additional controls. 
(i) PHF6 binds rDNA itself, and (ii) whether PHF6 binds to pre-rRNA coding or IGS sequences (Figure $3 \mathrm{c}$ ). It has long been recognized that the IGS contains multiple RNA Pol I responsive promoters, consistent with the identification of multiple non-coding IGS transcripts. ${ }^{34-36}$ Although the functions of most of these non-coding RNAs remain unknown, several have been implicated in direct silencing of rRNA transcription (eg, pRNAs), ${ }^{37,38}$ or of indirect silencing (eg, IGS ${ }_{16}$ RNA, $\mathrm{IGS}_{22} \mathrm{RNA}$, IGS ${ }_{28} \mathrm{RNA}$ ) by inducing the formation of a nucleolar detention center in response to environmental stress. ${ }^{39,40}$

As shown in Figure 3c, ChIP-qPCR for endogenous PHF6 demonstrated significant enrichment at the TSS and rDNA-coding sequences (primer sets $\mathrm{E}-\mathrm{H}$ ), with less binding at the promoter (primer set D), and no significant binding in the IGS (primer sets A-C) in comparison to IgG. To confirm the specificity of the assay, we also performed ChIP-qPCR for CTCF and UBF, both known to interact with the rDNA locus. ${ }^{41}$ Consistent with previous reports, CTCF was primarily enriched at the rDNA promoter, while UBF bound to the rDNA-coding sequence, with maximum enrichment at the $5^{\prime}$ end..$^{21,42}$ Histone $\mathrm{H} 3$ demonstrated strong enrichment throughout the repeat (Supplementary Figure S2C).

\section{PHF6 overexpression represses rRNA and activates non-coding IGS transcripts}

We next investigated whether PHF6 binding to rDNA-coding sequences enhanced or repressed rRNA expression by quantitating rRNA levels in a PHF6 gain- or loss-of-function model. To do so, we used cell lines overexpressing N- or C-terminally Flag-tagged PHF6 (PHF6-NTAP, PHF6-CTAP) ${ }^{10}$ and three different shRNAs for PHF6 knockdown. Overexpression or knockdown was confirmed at both the mRNA and protein levels (Supplementary Figures S3A-C). Although PHF6 knockdown in HeLa cells has been reported to reduce cell proliferation and induce $\mathrm{G}_{2} / \mathrm{M}$ arrest, ${ }^{14}$ we did not observe any such defects in the HEK 293T knockdown cells (data not shown).

To quantitate rRNA, overexpression and knockdown cell lines were seeded at a low confluency, and total RNA was isolated 2 days later for qRT-PCR analysis using the following primer sets (see Figure 3c): $28 \mathrm{~S}$ rRNA (primer sets $\mathrm{G}$ and $\mathrm{H}$ ), 47S pre-rRNA (F), unprocessed pRNA (D), IGS ${ }_{28}$ RNA (A), IGS ${ }_{36}$ RNA (B), and IGS $_{39}$ RNA (C). Consistent with previous studies, the $47 \mathrm{~S}$ pre-rRNA transcripts were $\sim 80$-fold higher and the $28 \mathrm{~S}$ rRNA levels are $\sim 10^{3}-10^{5}$ times more elevated than pRNA transcripts (Supplementary Figure S4A). ${ }^{37}$ When rRNA levels were quantitated in overexpression cell lines, a statistically significant decrease in the relative amount of $28 \mathrm{~S}$ rRNA was observed for primer set $\mathrm{G}$ (Figure 4a). Similarly, primer sets $\mathrm{F}$ and $\mathrm{H}$ showed a decrease but did not achieve statistical significance. Unexpectedly, we observed elevated levels of unprocessed pRNA, IGS ${ }_{36} \mathrm{RNA}$, and $\mathrm{IGS}_{39} \mathrm{RNA}$, with a significant reduction of $\mathrm{IGS}_{28} \mathrm{RNA}$. Indeed, the expression levels of $\mathrm{IGS}_{39} \mathrm{RNA}$ were almost equivalent to those of $47 \mathrm{~S}$ pre-rRNA in our overexpression cell lines (Supplementary Figure S4A). Although PHF6 knockdown also yielded an $\mathrm{IGS}_{28} \mathrm{RNA}$ decrease, no significant rRNA or IGS transcript level changes were observed for primer sets B-H (Supplementary Figure S4B). Despite these expression changes, we observed a PHF6-binding profile across the locus similar to wild-type cells (Supplementary Figures S3D and C). Taken together, increased PHF6 protein levels reduced rRNA expression and promoted an indirect activation of pRNA and IGS transcripts.

\section{Non-coding IGS transcripts are regulated by RNA Pol I}

PHF6 overexpression correlated with increased $\mathrm{IGS}_{36} \mathrm{RNA}$ and IGS $_{39}$ RNA expression (Figure 4a), but PHF6 does not bind to the
IGS (Figure 3c). Given that $\mathrm{IGS}_{36} \mathrm{RNA}$ and $\mathrm{IGS}_{39} \mathrm{RNA}$ are induced during transcriptional stress, ${ }^{39}$ we hypothesized that PHF6 overexpression might represent a source of such stress. ${ }^{14}$ To better understand the conditions by which $\mathrm{IGS}_{36} \mathrm{RNA}$ and $\mathrm{IGS}_{39} \mathrm{RNA}$ are expressed, we quantitated RNA from wild-type HEK 293T cells treated with chemical inhibitors of ribosome biogenesis (ActD, DRB), as well as TSA, a histone deacetylase inhibitor, and 5azaC, an analog of cytidine that does not undergo DNA methylation, both of which act to enhance rDNA transcription. ${ }^{43,44}$ As expected, 47S pre-rRNA was potently inhibited by ActD and unaffected by DRB (Figure $4 \mathrm{~b}$ ). ActD treatment also inhibited $\mathrm{IGS}_{36} \mathrm{RNA}$ and $\mathrm{IGS}_{39} \mathrm{RNA}$, indicating that they are likely transcribed by RNA Pol I. Conversely, DRB treatment, which uncouples rRNA transcription from rRNA processing to inhibit ribosome biogenesis, resulted in increased levels of $\mathrm{IGS}_{36} \mathrm{RNA}$ $(P<0.05)$ and $\mathrm{IGS}_{39}$ RNA $(P=0.06)$ expression, suggesting that this represents a stress response. Meanwhile, both TSA and 5azaC modestly enhanced pre-rRNA and modestly reduced the expression of IGS $_{36}$ RNA and IGS $_{39}$ RNA. Taken together, these data are consistent with a model whereby non-coding $\mathrm{IGS}_{36} \mathrm{RNA}$ and $\mathrm{IGS}_{39} \mathrm{RNA}$ are expressed in an RNA Pol I-dependent manner that is inversely proportional to the rate of pre-rRNA transcription (Figure 4c).

\section{DISCUSSION}

PHF6 is both nucleoplasmic and nucleolar; however, the literature provides sparse insight with respect to the contribution of nucleolar PHF6 toward inherited or acquired human disease. We now report that sub-nucleolar PHF6 is predominantly localized within the FC and DFC compartments, at whose interface rRNA transcription and early rRNA splicing occurs, in an RNA-dependent manner. Once localized, PHF6 is enriched at rDNA-coding sequences but not to IGS DNA sequences. Upon PHF6 overexpression, overall 47S pre-rRNA and $28 \mathrm{~S}$ rRNA levels decrease, coinciding with enhanced expression of repressive pre-pRNA and non-coding IGS RNA in a region approximately $3.5-7 \mathrm{~kb}$ upstream of the rDNA TSS.

To explain these results, we propose that, in addition to the reported role for PHF6 at the rDNA TSS (via its UBF interaction), PHF6 associates with its known interactor PAF1 during the transcriptional elongation of $47 \mathrm{~S}$ pre-rRNA (see Figure $4 \mathrm{c}$, top). ${ }^{13,14}$ RNA Pol I-mediated elongation of $47 \mathrm{~S}$ pre-rRNA is activated by PAF1 and is a key regulatory step during ribosome biogenesis that is coupled with the downstream mechanisms that process pre-rRNA into rRNA. ${ }^{23,45,46}$ Transcription along rDNA also requires a highly regulated structure to prevent the multiple polymerases that simultaneously occupy the same gene, as well as their associated interactions and nascent transcripts, from interfering with one another. ${ }^{21}$ As such, an overabundance of PHF6 may interfere with the rate of RNA Pol I elongation processivity by squelching PAF1, causing transcriptional stress that would lead to an arrest (Figure 4c, middle). As a result, RNA Pol I could become displaced from rDNA and instead begin transcribing IGS transcripts, such as $\mathrm{IGS}_{36} \mathrm{RNA}$ and $\mathrm{IGS}_{39} \mathrm{RNA}$, to further silence rDNA genes by promoting formation of a nucleolar detention center (Figure $4 \mathrm{c}$, bottom)..$^{39,40}$ Our ChIP-qPCR results support this proposed model, as they demonstrated strong enrichment of endogenous PHF6 protein at the TSS and across the entire gene body but not in the IGS. Moreover, these results are consistent with a recent ChIP-seq data set demonstrating that the majority of PHF6-binding sites occur in the gene bodies and proximal promoters of protein-coding genes. ${ }^{9}$ Although we would predict a similar result during PHF6 knockdown, we did not see this in our model, which may reflect the high genomic instability of HEK 293T cells that might favor ribosome production. ${ }^{47}$ 

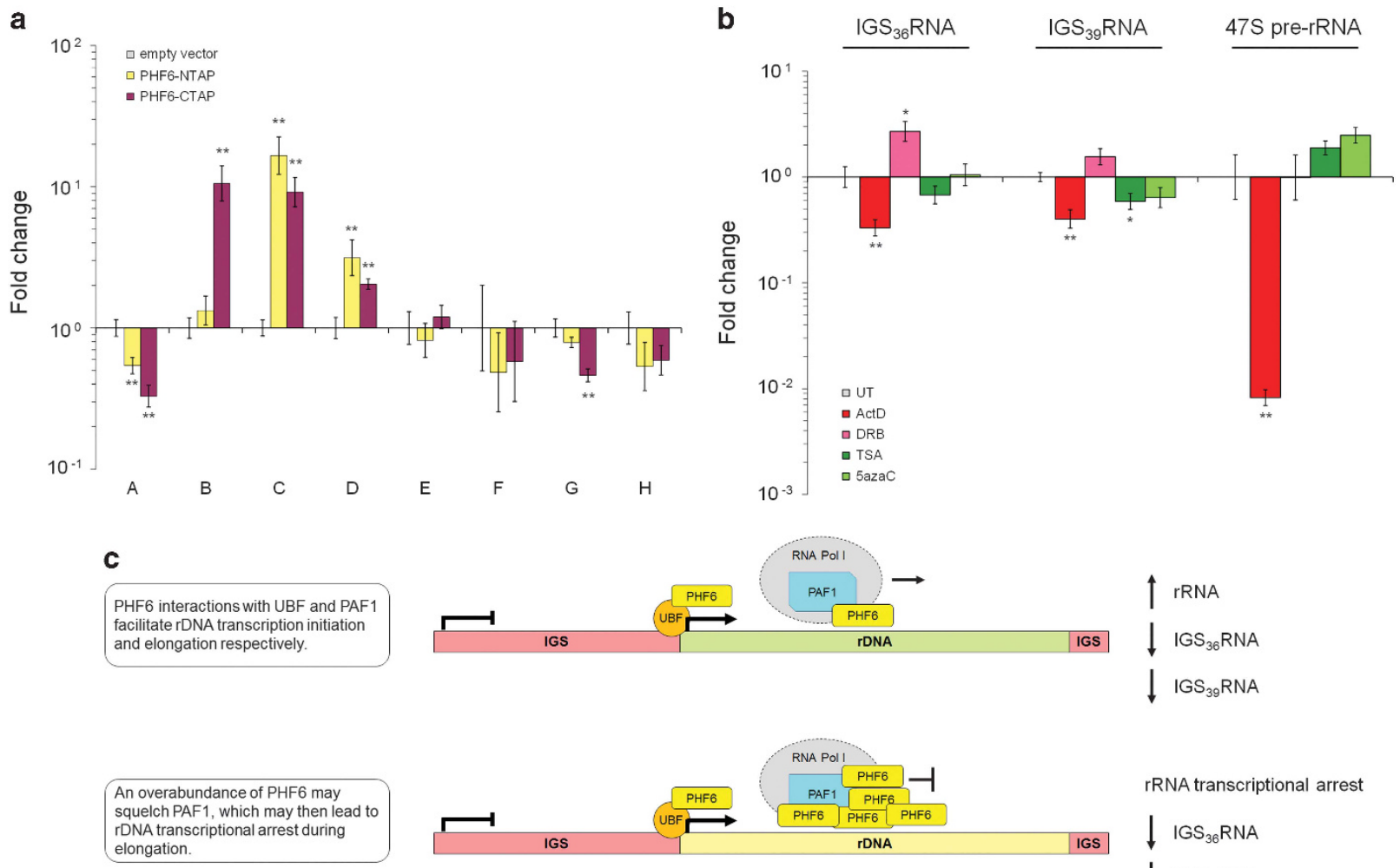

rRNA transcriptional arrest
$\downarrow \mathrm{IGS}_{36} \mathrm{RNA}$
$\downarrow \mathrm{IGS}_{39} \mathrm{RNA}$
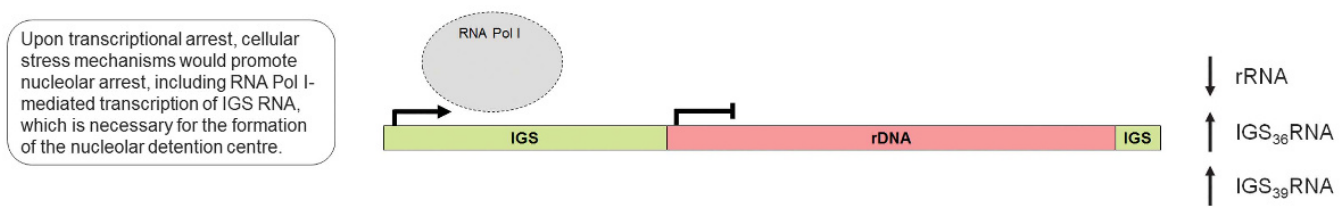

Figure 4 PHF6 mediates the expression of rDNA coding and non-coding transcripts. (a) qRT-PCR analysis $(n=8)$ of RNA isolated from HEK 293T cell lines expressing empty vector or one of two PHF6 overexpression constructs (PHF6-NTAP, PHF6-CTAP). All Ct values were normalized to GAPDH amplification and fold changes were calculated relative to the empty vector dCt value for all treatments. (b) qRT-PCR analysis $(n=6)$ of RNA isolated from wild-type HEK 293T cells that were treated with $0.5 \mu \mathrm{g} / \mathrm{ml}$ Act D $(2 \mathrm{~h}), 25 \mu \mathrm{g} / \mathrm{ml}$ DRB $(2 \mathrm{~h}), 10 \mathrm{ng} / \mathrm{ml}$ trichostatin A (24 h), or $50 \mu \mathrm{m} 5$-azacytidine $(24 \mathrm{~h})$. The $y$ axis is logarithmic (base 10). Bars represent SE ( ${ }^{*} P<0.05$, ${ }^{* *} P<0.01$, two-tailed Student's $t$-test). (c) Proposed model to explain how PHF6 overexpression could lead to the arrest of rRNA transcription and RNA Pol I-mediated expression of non-coding IGS transcripts. See Supplementary Figure S4 for additional controls.

In other models, however, PHF6 loss has been shown to impact transcription rates. ${ }^{13}$

Somewhat paradoxically, we observed that, despite its binding to the rDNA locus, the localization of PHF6 to the nucleolus was an RNAdependent process, implying that PHF6 requires an interaction with either non-coding RNA or perhaps even the nascent $47 \mathrm{~S}$ pre-rRNA to remain in the nucleolus and bound to its rDNA target. This finding is similar to observations for fibrillarin, which also localizes to the FC/ DFC boundary and associates with both rRNA and rDNA. ${ }^{32}$ As rRNA transcription and early processing events occur at the FC/DFC boundary, these data suggest that the ability of PHF6 to interact with both RNA and DNA may be critical for the regulation of ribosome biogenesis. As a potential explanation, PHF6 contains two ZaP nucleic acid interaction domains, and the second of these domains is known to bind dsDNA. ${ }^{11,48}$ Interestingly, missense changes within the first ZaP domain of PHF6 were shown to be essential for nucleolar localization, although this domain was proposed to be an interaction surface for UBF and thus indirect recruitment. ${ }^{14}$ Given that both $\mathrm{ZaP}$ domains of PHF6 share strong homology, we propose that PHF6 may interact with nascent pre-rRNA through its first ZaP domain, while binding to rDNA through its second ZaP domain. In this way, PHF6 may potentially act as a scaffold, bridging transcriptional elongation with early rRNA processing within the FC and DFC.

Abnormal nucleoli are a hallmark of several developmental and acquired human diseases, including intellectual disability and cancer. Dysfunctional ribosome biogenesis results from sequence variants that affect key functions of rDNA regulation, including epigenetic activation/silencing, the RNA Pol I transcriptional machinery, or the posttranscriptional processing of pre-ribosomes and maturing rRNA transcripts. ${ }^{17}$ We have provided evidence that PHF6 is localized to the FC/DFC boundary within the nucleolus where it is likely involved in the regulation of rRNA transcriptional elongation and early rRNA processing. Future efforts should be directed at defining its specific role in ribosome biogenesis and delineating how PHF6 variants promote disease pathogenesis.

\section{CONFLICT OF INTEREST}

The authors declare no conflict of interest. 


\section{ACKNOWLEDGEMENTS}

L Trinkle-Mulcahy, R Parks, K Yan, J Hamill, M Alvarez-Saavedra, D Ivanochko, and L Racacho provided reagents and/or technical advice. MAMT was supported by an Ontario Graduate Scholarship. This work was supported by operating grants to DJP from the Canadian Institutes of Health Research (MOP-97764, MOP-133586) and an operating grant from the Cancer Research Society, University of Ottawa, and Ottawa Hospital Research Institute partnership program.

1 Lower KM, Turner G, Kerr BA et al: Mutations in PHF6 are associated with BorjesonForssman-Lehmann syndrome. Nat Genet 2002; 32: 661-665.

2 Gecz J, Turner G, Nelson J, Partington M: The Borjeson-Forssman-Lehman syndrome (BFLS, MIM \#301900). Eur J Hum Genet 2006; 14: 1233-1237.

3 Van Vlierberghe P, Palomero T, Khiabanian $\mathrm{H}$ et al: PHF6 mutations in T-cell acute lymphoblastic leukemia. Nat Genet 2010; 42: 338-342.

4 Van Vlierberghe P, Patel J, Abdel-Wahab 0 et al: PHF6 mutations in adult acute myeloid leukemia. Leukemia 2011; 25: 130-134.

5 Zweier C, Rittinger O, Bader I et al: Females with de novo aberrations in PHF6: clinical overlap of Borjeson-Forssman-Lehmann with Coffin-Siris syndrome. Am J Med Genet C Semin Med Genet 2014; 166C: 290-301.

6 Chao MM, Todd MA, Kontny U et al: T-cell acute lymphoblastic leukemia in association with Borjeson-Forssman-Lehmann syndrome due to a mutation in PHF6. Pediatr Blood Cancer 2010; 55: 722-724.

7 Carter MT, Picketts DJ, Hunter AG, Graham GE: Further clinical delineation of the Borjeson-Forssman-Lehmann syndrome in patients with PHF6 mutations. Am J Med Genet A 2009; 149A: 246-250.

8 Todd MA, Ivanochko D, Picketts DJ: PHF6 degrees of separation: the multifaceted roles of a chromatin adaptor protein. Genes 2015; 6: 325-352.

9 Meacham CE, Lawton LN, Soto-Feliciano YM et al: A genome-scale in vivo loss-offunction screen identifies Phf6 as a lineage-specific regulator of leukemia cell growth. Genes Dev 2015; 29: 483-488.

10 Todd MA, Picketts DJ: PHF6 interacts with the nucleosome remodeling and deacetylation (NuRD) complex. J Proteome Res 2012; 11: 4326-4337.

11 Liu Z, Li F, Ruan K et al: Structural and functional insights into the human BorjesonForssman-Lehmann syndrome-associated protein PHF6. J Biol Chem 2014; 289: 10069-10083.

12 Zhang J, Jackson AF, Naito $T$ et al: Harnessing of the nucleosome-remodeling deacetylase complex controls lymphocyte development and prevents leukemogenesis. Nat Immunol 2012; 13: 86-94.

13 Zhang C, Mejia LA, Huang J et al: The X-linked intellectual disability protein PHF6 associates with the PAF1 complex and regulates neuronal migration in the mammalian brain. Neuron 2013; 78: 986-993.

14 Wang J, Leung JW, Gong Z, Feng L, Shi X, Chen J: PHF6 regulates cell cycle progression by suppressing ribosomal RNA synthesis. J Biol Chem 2013; 288 3174-3183.

15 Derenzini M, Montanaro L, Trere D: What the nucleolus says to a tumour pathologist. Histopathology 2009; 54: 753-762.

16 De Keersmaecker K, Sulima SO, Dinman JD: Ribosomopathies and the paradox of cellular hypo- to hyperproliferation. Blood 2015; 125: 1377-1382.

17 Hannan KM, Sanij E, Rothblum LI, Hannan RD, Pearson RB: Dysregulation of RNA polymerase I transcription during disease. Biochim Biophys Acta 2013; 1829 342-360.

18 Hetman M, Pietrzak M: Emerging roles of the neuronal nucleolus. Trends Neurosci 2012; 35: 305-314.

19 Tschochner H, Hurt E: Pre-ribosomes on the road from the nucleolus to the cytoplasm. Trends Cell Biol 2003; 13: 255-263.

20 Boisvert FM, van Koningsbruggen S, Navascues J, Lamond Al: The multifunctional nucleolus. Nat Rev Mol Cell Biol 2007; 8: 574-585.

21 Denissov S, Lessard F, Mayer $C$ et al: A model for the topology of active ribosomal RNA genes. EMBO Rep 2011; 12: 231-237.

22 Xie W, Ling T, Zhou Y et al: The chromatin remodeling complex NuRD establishes the poised state of rRNA genes characterized by bivalent histone modifications and altered nucleosome positions. Proc Natl Acad Sci USA 2012; 109: 8161-8166.

23 Zhang Y, ADt Smith, Renfrow MB, Schneider DA: The RNA polymerase-associated factor 1 complex (Paf1C) directly increases the elongation rate of RNA polymerase $\mathrm{I}$ and is required for efficient regulation of rRNA synthesis. J Biol Chem 2010; 285 $14152-14159$.
24 Chamousset D, De Wever V, Moorhead GB et al: RRP1B targets PP1 to mammalian cell nucleoli and is associated with Pre-60S ribosomal subunits. Mol Biol Cell 2010; 21 4212-4226.

25 Scheer U, Thiry M, Goessens G: Structure, function and assembly of the nucleolus. Trends Cell Biol 1993; 3: 236-241.

26 Weisenberger D, Scheer U: A possible mechanism for the inhibition of ribosomal RNA gene transcription during mitosis. J Cell Biol 1995; 129: 561-575.

27 Dundr M, Misteli T, Olson MO: The dynamics of postmitotic reassembly of the nucleolus. J Cell Biol 2000; 150: 433-446.

28 Scheer U, Hugle B, Hazan R, Rose KM: Drug-induced dispersal of transcribed rRNA genes and transcriptional products: immunolocalization and silver staining of different nucleolar components in rat cells treated with 5,6-dichloro-beta-D-ribofuranosylbenzimidazole. J Cell Biol 1984; 99: 672-679.

29 Zandomeni R, Zandomeni MC, Shugar D, Weinmann R: Casein kinase type II is involved in the inhibition by 5,6-dichloro-1-beta-D-ribofuranosylbenzimidazole of specific RNA polymerase II transcription. J Biol Chem 1986; 261: 3414-3419.

30 Lindell TJ, Weinberg F, Morris PW, Roeder RG, Rutter WJ: Specific inhibition of nuclear RNA polymerase II by alpha-amanitin. Science 1970; 170: 447-449.

31 Boulon S, Westman BJ, Hutten S, Boisvert FM, Lamond Al: The nucleolus under stress. Mol Cell 2010; 40: 216-227.

32 Ochs RL, Lischwe MA, Spohn WH, Busch H: Fibrillarin: a new protein of the nucleolus identified by autoimmune sera. Biol Cell 1985; 54: 123-133.

33 Zhu Z, Wang Y, Li X et al: PHF8 is a histone H3K9me2 demethylase regulating rRNA synthesis. Cell Res 2010; 20: 794-801.

34 Kuhn A, Grummt I: A novel promoter in the mouse rDNA spacer is active in vivo and in vitro. EMBO J 1987; 6: 3487-3492.

35 Cassidy BG, Yang-Yen HF, Rothblum LI: Additional RNA polymerase I initiation site within the nontranscribed spacer region of the rat rRNA gene. Mol Cell Biol 1987; 7: 2388-2396.

36 Jacob MD, Audas TE, Mullineux ST, Lee S: Where no RNA polymerase has gone before: novel functional transcripts derived from the ribosomal intergenic spacer. Nucleus 2012; 3: 315-319.

37 Mayer C, Schmitz KM, Li J, Grummt I, Santoro R: Intergenic transcripts regulate the epigenetic state of rRNA genes. Mol Cell 2006; 22: 351-361.

38 Schmitz KM, Mayer C, Postepska A, Grummt I: Interaction of noncoding RNA with the rDNA promoter mediates recruitment of DNMT3b and silencing of rRNA genes. Genes Dev 2010; 24: 2264-2269.

39 Audas TE, Jacob MD, Lee S: Immobilization of proteins in the nucleolus by ribosomal intergenic spacer noncoding RNA. Mol Cell 2012; 45: 147-157.

40 Jacob MD, Audas TE, Uniacke J, Trinkle-Mulcahy L, Lee S: Environmental cues induce a long noncoding RNA-dependent remodeling of the nucleolus. Mol Biol Cell 2013; 24 2943-2953.

41 Barski A, Cuddapah S, Cui $\mathrm{K}$ et al: High-resolution profiling of histone methylations in the human genome. Cell 2007; 129: 823-837.

42 van deNobelen S, Rosa-Garrido M, Leers J et al: CTCF regulates the local epigenetic state of ribosomal DNA repeats. Epigenetics Chromatin 2010; 3: 19.

43 Chen ZJ, Pikaard CS: Epigenetic silencing of RNA polymerase I transcription: a role for DNA methylation and histone modification in nucleolar dominance. Genes Dev 1997; 11: 2124-2136.

44 Santoro R, Grummt I: Molecular mechanisms mediating methylation-dependent silencing of ribosomal gene transcription. Mol Cell 2001; 8: 719-725.

45 Schneider DA, Michel A, Sikes ML et al: Transcription elongation by RNA polymerase I is linked to efficient rRNA processing and ribosome assembly. Mol Cell 2007; 26: 217-229.

46 Yang $Y$, Li W, Hoque $M$ et al: PAF complex plays novel subunit-specific roles in alternative cleavage and polyadenylation. PLoS Genet 2016; 12: e1005794.

47 Lin YC, Boone M, Meuris L et al: Genome dynamics of the human embryonic kidney 93 lineage in response to cell biology manipulations. Nat Commun 2014: 5: 4767.

48 Liu L, Qin S, Zhang J, Ji P, Shi Y, Wu J: Solution structure of an atypical PHD finger in BRPF2 and its interaction with DNA. J Struct Biol 2012; 180: 165-173.

(c) (1) (2) This work is licensed under a Creative Commons ar no sA Attribution-NonCommercial-ShareAlike 4.0 International License. The images or other third party material in this article are included in the article's Creative Commons license, unless indicated otherwise in the credit line; if the material is not included under the Creative Commons license, users will need to obtain permission from the license holder to reproduce the material. To view a copy of this license, visit http:// creativecommons.org/licenses/by-nc-sa/4.0/

Supplementary Information accompanies this paper on European Journal of Human Genetics website (http://www.nature.com/ejhg) 